VOL. 1, NO. 2

\title{
Local Heat-Transfer Performance and Mechanisms in Radial Flow Between Parallel Disks
}

\author{
S. Mochizuki* \\ Tokyo University of Agriculture and Technology, Tokyo, Japan \\ and \\ Wen-Jei Yang $\dagger$ \\ University of Michigan, Ann Arbor, Michigan
}

\begin{abstract}
An experimental study is conducted to determine local heat-transfer performance and mechanisms in radial flow through two parallel heated disks. Three distinct heat-transfer mechanisms are disclosed along the flow passage: steady laminar, periodic laminar, and turbulent. The heat-transfer performance is enhanced proportional to the 0.5 and 0.8 powers of Reynolds number, in the steady periodic and turbulent ranges, respectively. The local and average heat-flow maps are constructed to define the domain or range of the transfer mechanisms. The locations for the onset of both steady laminar and turbulent mechanisms shift upstream with an increase in the flow rate.
\end{abstract}

\section{Nomenclature}

$a \quad=s / 2, \mathrm{~m}$

$D_{H} \quad=$ hydraulic diameter, $\mathrm{m} ;=2 s$

$d \quad=$ disk diameter, $\mathrm{m}$

$d_{1}, d_{2} \quad=$ inner and outer diameter, respectively $=\left(d_{1}+d_{2}\right) / 2$

$G z^{*} \quad=$ modified Graetz number defined as $\mathrm{Pe} d_{m} / D_{H}$

$h \quad=$ local heat-transfer coefficient, $\mathrm{W} / \mathrm{m}^{2}-{ }^{\circ} \mathrm{C}$

$h_{m} \quad=$ average value

$k^{m}=$ thermal conductivity of fluid, $\mathrm{W} / \mathrm{m}-{ }^{\circ} \mathrm{C}$

$\mathrm{Nu} \quad=$ local Nusselt number

$N u_{m} \quad=$ average value

$P e^{m} \quad=$ Peclet number

$\dot{q} \quad=$ local heat flux, $\mathrm{W} / \mathrm{m}^{2}$

$\dot{q}^{\prime} \quad=$ value of $\dot{q}$ being corrected for heat loss

Re $\quad=$ Reynolds number

$R e_{C}, R e_{t}=$ critical and transition values, respectively

$R, R_{1}, R_{2}=r / a, r_{1} / a$, and $r_{2} / a$, respectively

$r \quad=$ radial distance, $m$

$r_{1}, r_{2}=$ inner and outer radii, respectively

$r_{m} \quad=\left(r_{1}+r_{2}\right) / 2$

$s \quad=$ disk spacing, $\mathrm{m}$

$T \quad=$ temperature, ${ }^{\circ} \mathrm{C}$

$T_{a}, T_{w} \quad=$ temperature of air and disk surface, respectively

$U_{m}$

$U_{m}$
$X$

$=$ mean flow velocity at $r_{m}, \mathrm{~m} / \mathrm{s}$

= electrical power input, $\mathbf{W}$

$=$ dimensionless radial distance

$=$ kinematic viscosity, $\mathrm{m}^{2} / \mathrm{s}$

Subscripts

\begin{tabular}{|c|c|}
\hline$a$ & $=$ fluid \\
\hline$m$ & $=$ midchannel \\
\hline$W$ & $=$ surface \\
\hline 1 & $=$ inlet \\
\hline 2 & $=$ exit \\
\hline
\end{tabular}

Received Feb. 3, 1986; revision received May 12, 1986. Copyright (C) American Institute of Aeronautics and Astronautics, Inc., 1986. All rights reserved.

*Associate Professor, Department of Mechanical Engineering.

†Professor, Department of Mechanical Engineering and Applied Mechanics.

\section{Introduction}

$\mathbf{O}$ NE of the basic flow systems is a radial flow through a pair of parallel circular disks. It is an extension of a channel flow between two parallel planes. There are, however, two important differences between the two flow systems. Both the flow cross-sectional area and the pressure drop are constant along the flow passage in the channel flow system. In the case of the radial flow system, the flow cross-sectional area increases along the flow passage, while the pressure gradient changes from negative in the entrance region to positive in the downstream region. Accordingly, it is conceivable that a flow separation region is created at the location of transition in the pressure gradients from a negative to positive value. Therefore, in contrast to a developing (i.e., entrance) flow followed by a fully developed flow in the channel flow case, a more complex phenomenon is anticipated in the radial flow system.

Based on the finite-difference solutions of the steady vorticity transport equation, Raal $^{1}$ concluded that, above a certain value of Reynolds number $(R e)$, separation is observed, with the bubble size increasing rapidly with $R e$. Mochizuki and Yang $^{2}$ studied the radial flow behavior by means of the flow visualization methods of dye injection, hydrogen-bubble generation, and paraffin mist. Three distinct flow patterns were observed:

1) Laminar flow for $R e<R e_{c}$.

2) A self-controlled flow oscillation that decays further downstream, in the range of $R e_{c} \leq R e \leq R e_{t}$.

3) Turbulent flow with a reverse transition further downstream, when $R e \leq R e_{t}$.

$R e_{c}$ and $R e_{t}$ are the critical and transition Reynolds numbers, respectively. Mochizuki and Yang found that the oscillating flows were caused by a vortex street consisting of vortices that separated periodically and alternately from both disks. Finitedifference solutions of the unsteady vorticity transport equation generally agreed with certain experimental observations.

Only a few studies dealt with heat-transfer problems in the radial flow system: Kreith $^{3}$ investigated heat transfer in a fully developed flow. Using the unsteady test method by condensing steam inside hollow disks, Mochizuki and Yang ${ }^{4}$ studied the same system including the entrance effects. Suryanarayana et al. ${ }^{5}$ performed an experimental study on the heat-transfer performance of stationary and corotating parallel disks that were electrically heated. Each disk was concentrically divided into seven sections along the flow for separate heating. The 
"local" (referring to the sectional average value) heat-transfer performance exhibited a minimum at certain locations downstream from the entrance. No attempt was made to examine the cause of such a behavior.

The efforts of these researchers ${ }^{3-5}$ were focused on the overall (namely, average) heat-transfer performance in the radial flow system without considering the local variations of flow and heat-transfer mechanisms along the radial flow passage. In reference to the existence of various flow regimes, Mochizuki et al. ${ }^{6}$ performed an experimental study on heat transfer and friction loss in multiple parallel disk assemblies using a modified single-blow transient test method. Through a correlation of the average Nusselt number vs the Reynolds number, evidence displayed that the heat-transfer performance (although based on the overall behavior throughout the entire flow channel) indeed exhibited characteristics of different convective mechnisms dependent on the flow regimes. A schematic flow map was constructed to demonstrate the variation of flow patterns along the radial flow passage with the Reynolds number. From the correlations of the heattransfer and Fanning friction factors vs the Reynolds number, three distinct mechanisms of convective enhancement were found: the laminar-flow, "second" laminar-flow, and transition-turbulent flow enhancement. These are caused by inertia effects, vortex-induced flow oscillations, and random mixing, respectively. The major shortcomings of Refs. 6 and 7 are:

1) The radial flow system consists of multiple flow passages rather than a single channel.

2) Heat-transfer mechanisms are determined by the average $\mathrm{Nu}$ vs $\mathrm{Re}$ correlations rather than by local performance.

The present study aims to correct the two defects. An experimental study is conducted to determine the local heattransfer performance in the radial flow system. The local $N u$ vs $R e$ correlations disclose the distinct flow regimes and heattransfer mechanisms and determine the location of transitions between flow regimes. A flow map is constructed to define the flow regimes.

The results can be applied to the design of hydrostatic air bearings, centrifugal compressor diffusers, and radial-flowtype heat exchangers.

\section{Experimental Apparatus and Procedures}

The heat-transfer test section consisted of a pair of parallel circular disks placed horizontally with a disk spacing $s$, as shown in Fig. 1. Each disk was made of Bakelite $(10 \mathrm{~mm}$ thick with $160-\mathrm{mm}$ i.d. and $500-\mathrm{mm}$ o.d.) attached with an electrically conductive plastic film as a heat-generating element. The plastic film was $0.2 \mathrm{~mm}$ in thickness, of which $0.02 \mathrm{~mm}$ was an electrically conducting layer having uniform electrical resistance. It is commonly used in potentiometers. The ringtype electrodes were attached to both the inner and outer rims of the plastic films. By passing an electrical current, heat was generated in the conducting layers, thus turning the exposed side of each plastic film into a heating surface. The local heat flux $\dot{q}$ at a radial distance $r$ varies as

$$
\dot{q}=W /\left[2 \pi r^{2} \ln \left(r_{2} / r_{1}\right)\right]
$$

The electrical power imposed on each disk, $W$, is constant. Equation (1) takes into account the local variation of electrical resistance.

The distribution of heating-surface temperatures $T_{w}$ was measured by means of $24(0.0025$-mm-diam) Alumel-Chromel thermocouples. Figure 2 shows the installation of a heatingsurface thermocouple. The thermocouple was inserted through a tiny hole in the plastic film and attached to the inner surface of the conductive layer. The hole was then filled with a heat-resistant adhesive agent. Both the upper and lower disks were externally insulated by styrene foam layers on the back side and external circumference of each Bakelite layer.

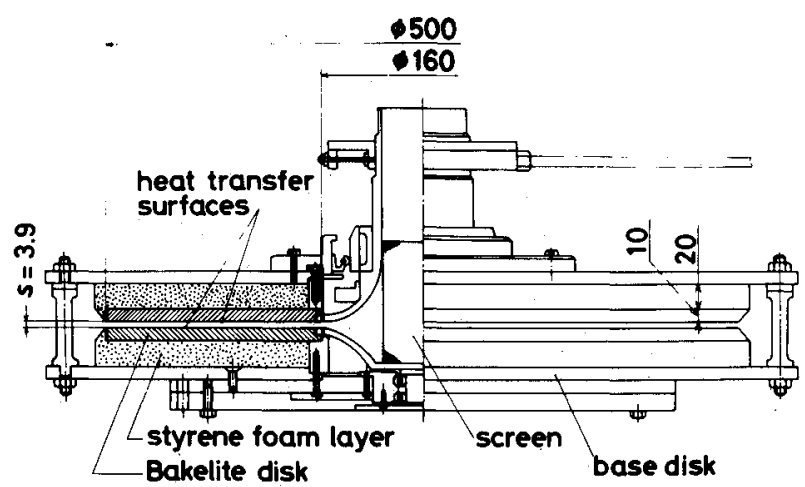

Fig. 1 Heat-transfer test section.

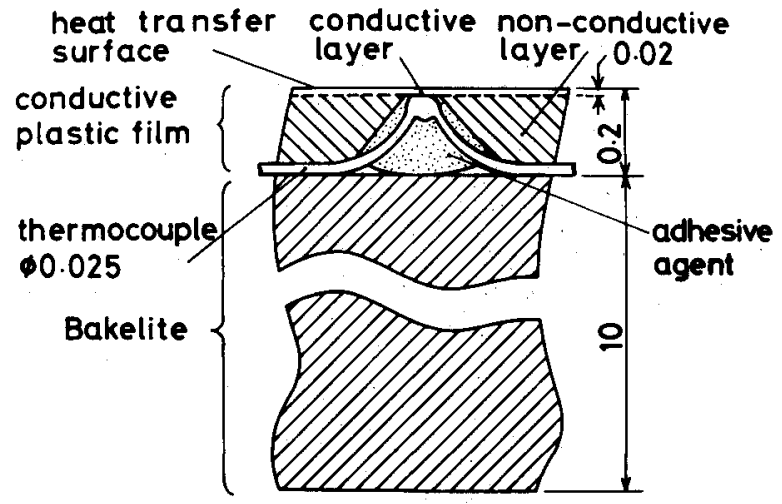

Fig. 2 Installation of heating-surface thermocouples.

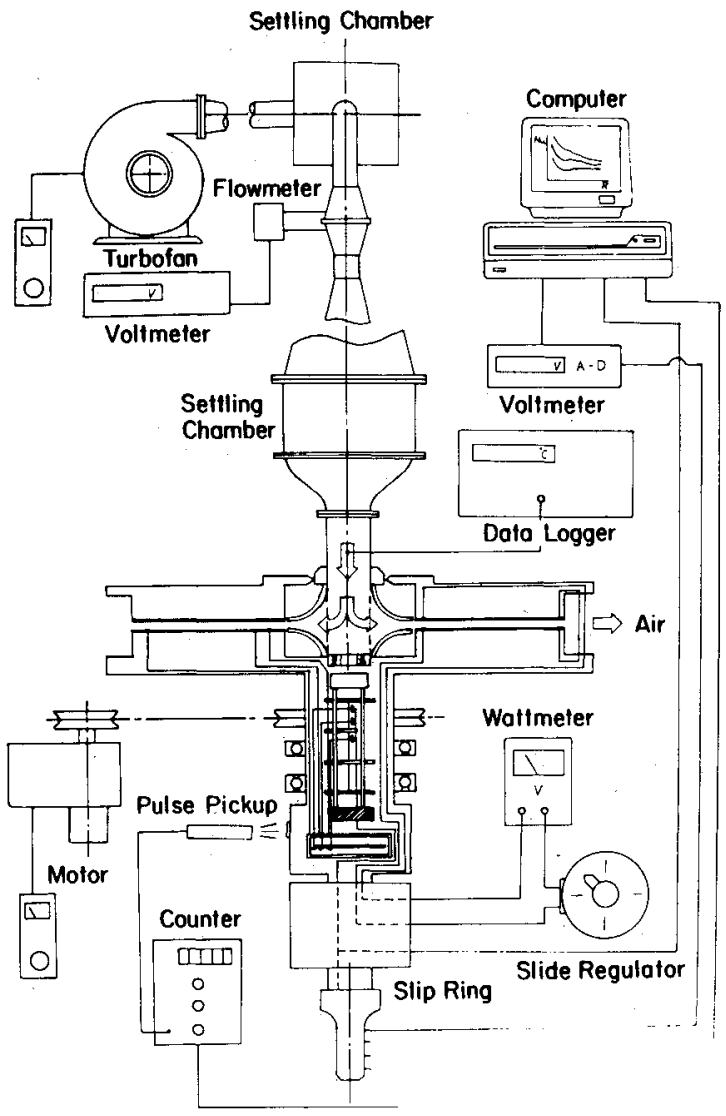

Fig. 3 Schematic of experimental apparatus. 
The entire experimental setup is schematically illustrated in Fig. 3. Air was supplied to the heat-transfer test section from the turbofan through a settling chamber. The airflow rate was monitored by a flowmeter and read from the voltmeter. After altering its flow direction to pass through a screen and bell mouth, the air entered into the radial flow channel, being heated on its way out. A 0.025 -mm-diam Alumel-Chromel thermocouple was installed at the entrance of the heat-transfer test section to measure the inlet air temperature. All measured data were fed into a microcomputer for processing. A hot wire was employed to check the azimuthal uniformity of flow.

\section{Test Results and Discussion}

The test section had the physical dimensions $r_{1}=80 \mathrm{~mm}$, $r_{2}=217 \mathrm{~mm}$, and $s=3.9 \mathrm{~mm}$. Hence, $d_{i}=160 \mathrm{~mm}$, $d_{2}=434 \mathrm{~mm}$, and $d_{1} / S=41$. The flow was varied from $R e=400-12,000$. Here, $R e$ is defined as

$$
\operatorname{Re}=u_{m} D_{H} / \nu
$$

Figure 4 is a typical result, showing the distributions of heat flux $\dot{q}$, surface temperature $T_{w}$, and air temperature $T_{a}$ at $R e=2370 . T_{w}$ was measured while $\dot{q}$ was calculated by Eq. (1). $\dot{q}^{\prime}$ was the value of $\dot{q}$ being corrected for heat loss through the Bakelite and styrene foam plates to the steel support, assuming one-dimensional heat flow. The latter quantity represents the actual value communicated to the air. $T_{a}$ is the mixed mean gas temperature calculated using $\dot{q}^{\prime}$. The local heattransfer coefficient was determined by

$$
h=\dot{q}^{\prime} /\left(T_{w}-T_{a}\right)
$$

The local Nusselt number is defined as

$$
N u=h D_{H} / k
$$

The distribution of the local Nusselt number is given in Fig. 5 as a function of $R e$. Figure 5 is replotted for $N u$ vs $X$ in Fig. 6. $X$ is defined as

$$
X=4\left(s / d_{1}\right)\left(R^{2}-R_{1}^{2}\right) /\left[\operatorname{Pe}\left(1+d_{1} / d_{1}\right)\right]=\left(R^{2}-R_{1}^{2}\right) / G z^{*}(5)
$$

where $G z^{*}$ denotes the modified Graetz number. Two solid lines were superimposed in Fig. 6. Both are theoretical results

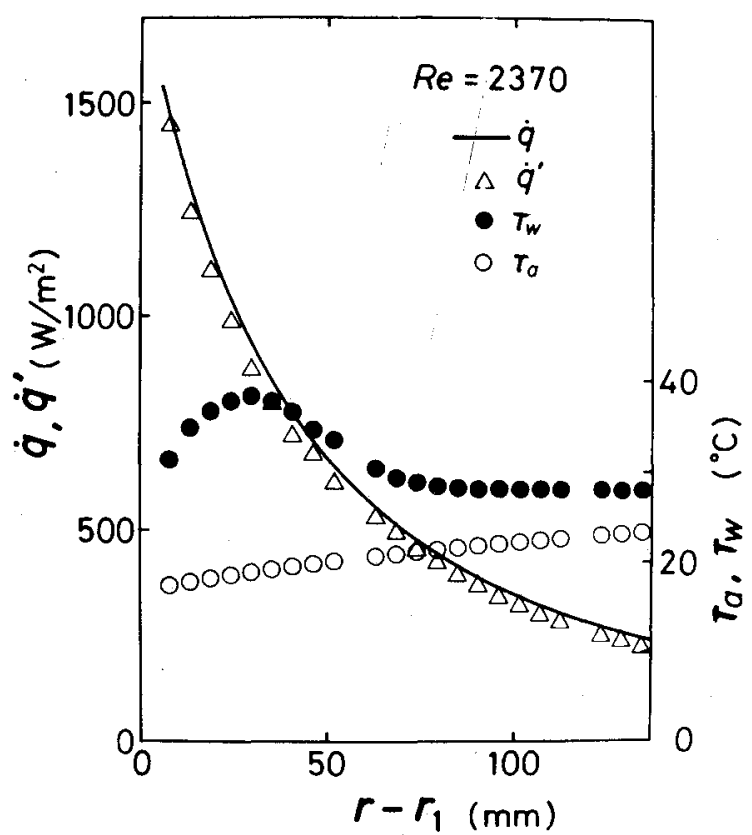

Fig. 4 Distribution of calculated and corrected heat fluxes, measured surface temperature, and calculated mean gas temperature. assuming a constant-velocity profile from the inlet to the exit, one with a uniform velocity profile as indicated by "slug flow" and the other with a parabolic velocity distribution as indicated by "Poiseuille flow." In both cases, the local Nusselt number is independent of $R e$.

An examination of Figs. 5 and 6 leads to the following observations:

1) When $R e$ is small, less than a critical value $R e_{c}, N u$ decreases monotonically with an increase in $\left(R-R_{1}\right)$ since the flow velocity diminishes in direct proportion to $\left(R-R_{1}\right)$. It reduces to slightly below the Poiseuille-flow value and then asymptotically approaches the Poiseuille limit as the velocity profile develops into a parabolic shape from a flat one at the entrance. During the course of the boundary-layer development, points of inflection occur in some velocity profiles. ${ }^{2}$ This is a singular feature of radial flow that cannot be observed in pipe flows. The value of $R e_{c}$ is $1000-1400$ in Fig. 5, but a hot-wire measurement indicated $R e=1200$ for the onset of boundary-layer separation. The range of $R e \leq R e_{c}$ is the steady flow regime without boundary-layer separation and reattachment.

2) In the range of $R e_{c} \leq R e \leq R e_{t}, N u$ reduces monotonically with $\left(R-R_{1}\right)$, while the flow is steady and laminar. It reaches the minimum at a certain value of $\left(R=R_{1}\right)$ and then rebounds. The rebounding of $N u$ is caused by the occurrence of an oscillating flow triggered by a vortex street consisting of vortices (i.e., separating annular bubbles). It was disclosed in Ref. 2 that a self-sustained sequence of nucleation, growth, migration, and decay of the vortices takes place in the radial flow between parallel disks induced by shear-layer separation and reattachment. The locus of the minimum values of $N u$ is indicated by the broken line in Fig. 5. It signifies that the location of the minimum $\mathrm{Nu}$ moves upstream with an increase in $R e$. The value of $R e_{t}$ is approximately 5000 . In the range of $R e_{c} \leq R e \leq R e_{t}$, the flow is steady, followed by a self-sustained fluctuation, which decays downstream.

3) When $R e$ exceeds $R e_{t}$, two minimum values are observed in the radial distribution of $N u$. The second minimum occurs due to flow transition from a laminar oscillating pattern to a turbulent type. The second rebounding of the $\mathrm{Nu}$ curve reflects turbulent heat-transfer performance. A reverse transition (i.e., relaminarization) takes place further downstream. ${ }^{2}$

Figure 7 is a plot of $N u$ vs $R e$ at three locations to confirm the existence of the three distinct patterns and their respective

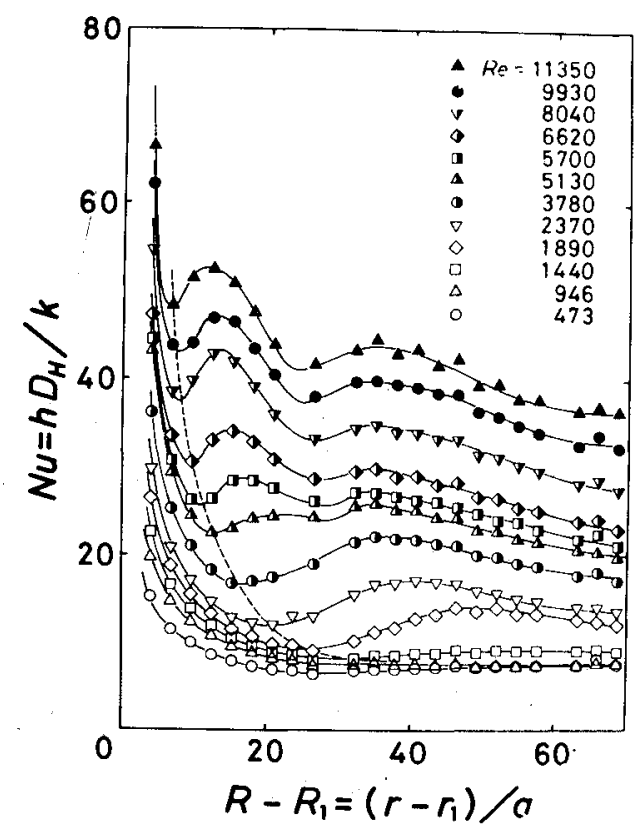

Fig. 5 Distribution of local Nusselt number $(N u$ vs $R-R)$ as a function of Reynolds number. 


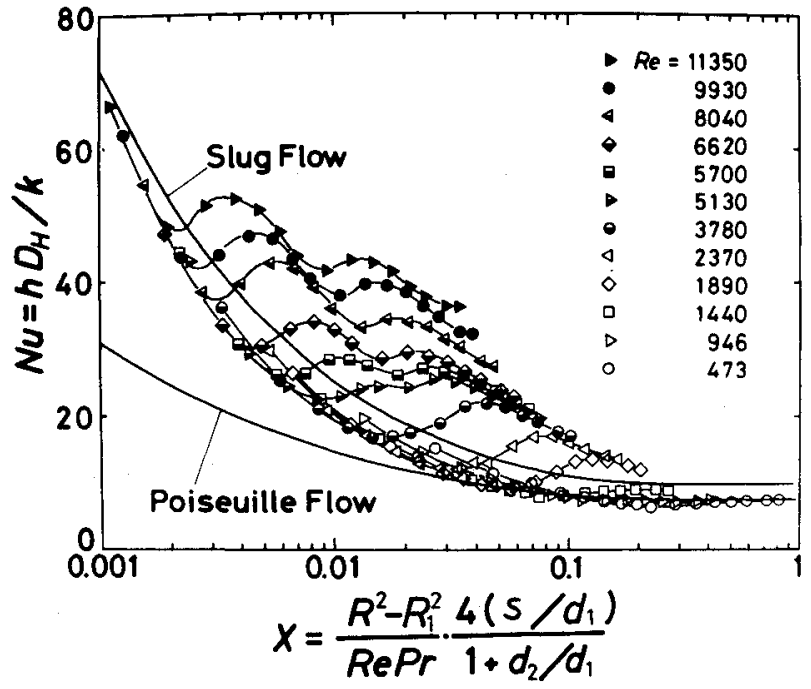

Fig. 6 Distribution of local Nusselt number $(\mathrm{Nu}$ vs $\mathrm{X})$ as a function of Reynolds number.

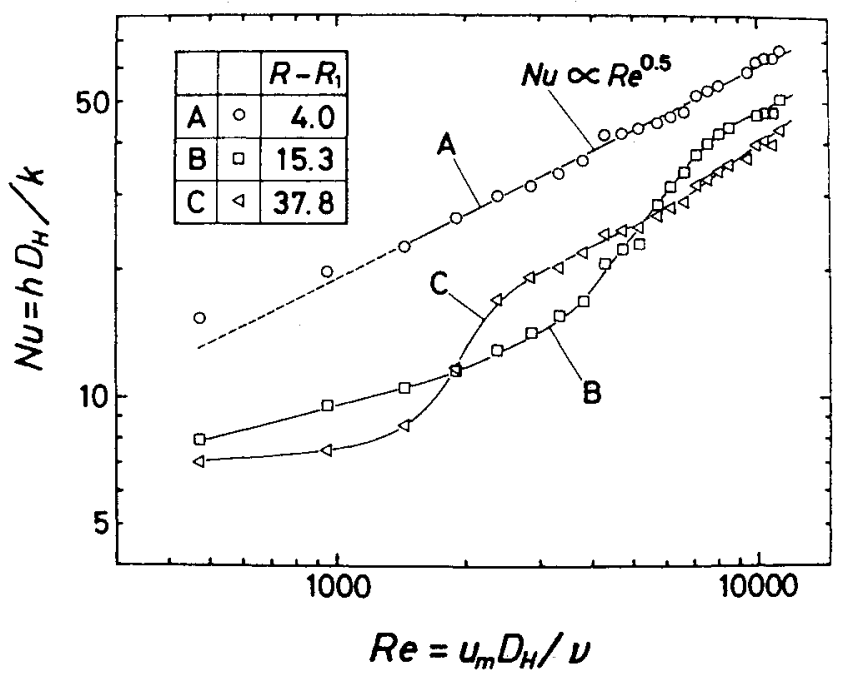

Fig. 7 Dependence of local Nusselt number on Reynolds number at various radial locations.

heat-transfer characteristics in radial flow between two parallel disks. At location $\mathrm{A}$ in the entrance region, $N u$ is proportional to $R e^{0.5}$, typical of laminar heat-transfer performance. At locations B (immediately downstream from the entrance region) and $\mathrm{C}$ (approximately midway in the channel), heat transfer exhibits a laminar behavior at low values of $R e$. However, when $R e$ exceeds a critical value, $N u$ increases sharply as a consequence of boundary-layer oscillations. The critical $R e$ for the onset of a sharp rise in $N u$ varies depending on the radial location because the boundary-layer separation point shifts upstream with an increase in $R e$. Figure 8 is a replot of the broken line in Fig. 5 in the form of $R e$ vs $\left(R-R_{1}\right)$. It represents the local heat-flow map defining the domains of steady laminar, periodic laminar, and turbulent mechanisms for the present system. One observes that

1) Laminar boundary-layer separation occurs at $R e$ of approximately 1200, referring to the broken line in Fig. 7.

2) The locations of both $R e_{c}$ and $R e_{t}$ shift upstream with an increase in $R e$.

The average Nusselt number over the entire test section is defined as

$$
h_{m}=\int_{r_{1}}^{r_{2}} 2 h r \mathrm{~d} r /\left(r_{2}^{2}-r_{1}^{2}\right)
$$

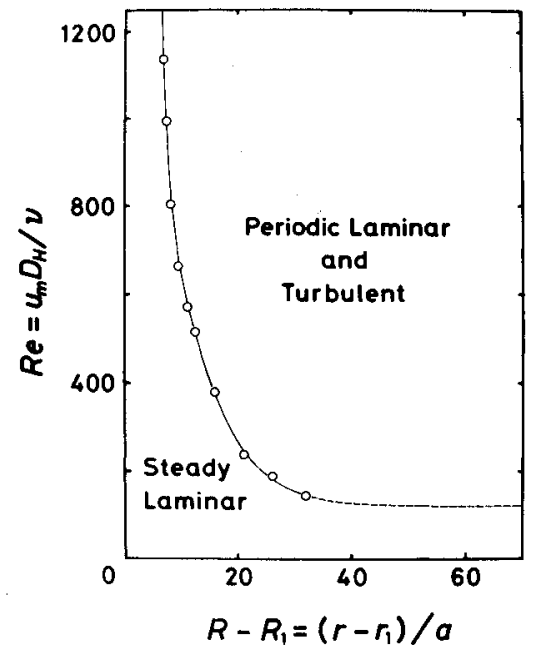

Fig. 8 Flow map defining flow regimes.

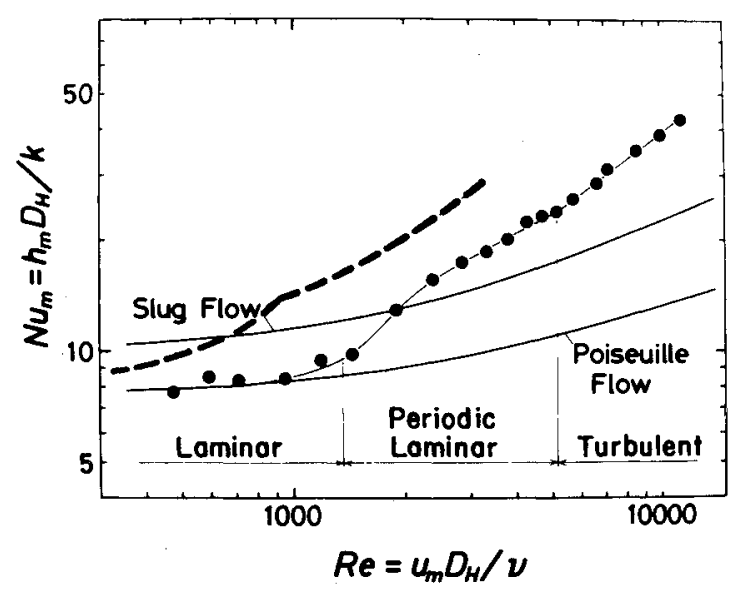

Fig. 9 Dependence of average Nusselt number on Reynolds number.

Figure 9 illustrates the $N u_{m}$ vs $R e$ relationship. At low values of $R e$, below $1200, N u_{m}$ is close to the theoretical result for Poiseuille flow. $N u_{m}$ increases sharply at higher values of $R e$. When $R e$ exceeds $5000, N u_{m}$ changes in direct proportion to $R e^{0.8}$, which is typical of turbulent characteristics. Figure 9 can be regarded as the overall heat-flow map defining the ranges of three distinct heat-transfer mechanisms in the radial flow through two parallel disks. The result for a multiple-disk assembly with condensation heating is superimposed in Fig. 9 (broken line) for comparison. For the multiple-disk case, the values of $N u_{m}$ are higher, and the onset of the periodic laminar and turbulent flows occurs at much lower Reynolds numbers. The uncertainty in $N u$ was estimated to be about $6 \%$.

\section{Conclusions}

Convective heat transfer between the electrically heated disk walls and the air in radial flow is experimentally investigated. The following conclusions have been derived from the study:

1) Both the local and average heat-transfer characteristics indicate the existence of three distinct heat-transfer mechanisms, namely, steady laminar, periodic laminar, and turbulent, depending on the flow rate.

2) $N u$ varies in direct proportion to $R e^{0.5}$ and $R e^{0.8}$ in the steady laminar and turbulent flow regimes, respectively.

3) The local flow map, an $R e$ vs $\left(R-R_{1}\right)$ plot, is constructed to define the domains for different mechanisms.

4) The overall heat-flow map, a $N u_{m}$ vs $R e$ plot, is constructed to define the ranges of $R e$ for different mechanisms. 
5) The locations for onset of both the periodic laminar and turbulent flows move upsteam as the flow is increased.

The above conclusions should be applicable to the general thermal situation. It should be noted that the uncertainty associated with the determination of the laminar/turbulent transition and reverse transition based on heat-transfer results is as much as the uncertainty associated with the determination of convective heat-transfer coefficients.

\section{References}

${ }^{1}$ Raal, J.D., "Radial Source Flow Between Parallel Disks," Journal of Fluid Mechanics, Vol. 85, 1978, pp. 401-416.

${ }^{2}$ Mochizuki, S. and Yang, W.J., "Self-Sustained Radial Oscillating Flows Between Parallel Disks," Journal of Fluid Mechanics, Vol. 154, 1985, pp. 377-397.

${ }^{3}$ Kreith, F. "Transfert de Chaleur et de Masse dans un Ecoulement Radial entre Deux Disques Paralléles Fixes, ou Tournant á la Meme
Vitesse," International Journal of Heat and Mass Transfer, Vol. 9, Feb. 1966, pp. 265-282.

${ }^{4}$ Mochizuki, S. and Yang, W.-J., "Heat Transfer and Friction Loss in Laminar Radial Flows through Rotating Annular Disks," Journal of Heat Transfer, Vol. 103, Aug. 1981, pp. 212-217.

${ }^{5}$ Suryanarayana, N.V., Scofield, T., and Kleiss, R.E., "Heat Transfer to a Fluid in Radial, Outward Flow Between Two Coaxial Stationary or Corotating Disks," Journal of Heat Transfer, Vol. 105, Aug. 1983, pp. 519-526.

${ }^{6}$ Mochizuki, S., Yang, W.-J., Yagi Y., and Ueno, M. "Heat Transfer Mechanisms and Performance in Multiple Parallel Disk Assemblies," Journal of Heat Transfer, Vol. 105, Aug. 1983, pp. 598-604.

${ }^{7}$ Mochizuki, S. and Yang, W.-J., "Three Mechanisms of Convective Enhancement in Stationary Disk Systems,"' to be published in Journal of Heat Transfer, 1986.

${ }^{8}$ Kline, S.J. and McClintock, F.A., "Describing Uncertainty in Single Sample Experiments," Mechanical Engineering, Vol. 75, Jan. 1953, pp. 3-8.

\title{
From the AIAA Progress in Astronautics and Aeronautics Series
}

\section{SPACECRAFT RADIATIVE TRANSFER AND TEMPERATURE CONTROL-v. 83}

\author{
Edited by T.E. Horton, The University of Mississippi
}

Thermophysics denotes a blend of the classical engineering sciences of heat transfer, fluid mechanics, materials, and electromagnetic theory with the microphysical sciences of solid state, physical optics, and atomic and molecular dynamics. This volume is devoted to the science and technology of spacecraft thermal control, and as such it is dominated by the topic of radiative transfer. The thermal performance of a system in space depends upon the radiative interaction between external surfaces and the external environment (space, exhaust plumes, the sun) and upon the management of energy exchange between components within the spacecraft environment. An interesting future complexity in such an exchange is represented by the recent development of the Space Shuttle and its planned use in constructing large structures (extended platforms) in space. Unlike today's enclosed-type spacecraft; these large structures will consist of open-type lattice networks involving large numbers of thermally interacting elements. These new systems will present the thermophysicist with new problems in terms of materials, their thermophysical properties, their radiative surface characteristics, questions of gradual radiative surface changes, etc. However, the greatest challenge may well lie in the area of information processing. The design and optimization of such complex systems will call not only for basic knowledge in thermophysics, but also for the effective and innovative use of computers. The papers in this volume are devoted to the topics that underlie such present and future systems.

Published in 1982, 529 pp., $6 \times 9$, illus., $\$ 35.00 \mathrm{Mem} ., \$ 55.00$ List 